\title{
Intensification of biokinetics of enzymes using ultrasound assisted methods: A critical review
}

\author{
Altab Khan ${ }^{1}$, Mohd Riyaz Beg ${ }^{2 *}$, Pramod Waghmare ${ }^{2}$
}

1. DBT-ICT Centre for Energy Biosciences, Institute of Chemical Technology, Mumbai 400019, Maharashtra, India

2. Department of Pharmaceutical Sciences and Technology, Institute of Chemical Technology, Mumbai 400019, Maharashtra, India

*Corresponding Author: Mohd Riyaz Beg

Address: Dept. of Pharm. Sci. \& Tech., Institute of Chemical Technology, Mumbai 400019, Maharashtra, India

Email: mohdriyazbeg@gmail.com

\begin{abstract}
The use of low intensity ultrasound has gotten surprising consideration over the last decade as a method for enhancing the catalytic activity of enzyme. Ultrasounds have the potential to significantly influence the activity of the enzymatic processes, provided that the energy input is not too high to inactivate the enzyme. By providing the variation in parameters, various physical and chemical effects can be attained that can enhance the enzymatic reaction. Ultrasonic reactors are known for their application in bioprocesses. However, the potential of their applications is still limited broadly due to the lack of proper information about their operational and performance parameters. In this review, the detailed information about ultrasonic reactors is provided by defining the different types of reactors, number and position of ultrasonic transducers. Also, it includes mechanism of intensification and influence of ultrasonic parameters (intensity, duty cycle and frequency) and enzymatic factors (enzyme concentration, temperature and $\mathrm{pH}$ ) on the catalytic activity of enzyme during ultrasound treatment.
\end{abstract}

Keywords: Ultrasound, Ultrasonic reactors, Enzyme kinetic, Enzymatic reaction, Sonochemical reaction 


\section{Introduction}

The rapid growth in biotechnology is continually attracting new strategies and solutions for further advancement of bioprocess performances. Ultrasound has gotten surprising consideration over the last decade as a rapid method for enhancing the efficiency of bioprocesses [1]. Ultrasonication is a branch of acoustics that can be applied to solids, liquids and gases at frequencies above the human hearing range. Ultrasound is defined as a sound wave having a frequency that exceeds the human ear's hearing limit $(20 \mathrm{kHz})$. There are two major types of ultrasound i.e., low \& high intensity. High intensity ultrasound uses high power $\left(>1 \mathrm{Wcm}^{-2}\right)$ and low frequency $(<0.1 \mathrm{MHz})$ whereas low intensity ultrasound uses low power $\left(<0.1 \mathrm{Wcm}^{-2}\right)$ and high frequency (0.1 to $100 \mathrm{MHz})$ [2].

Ultrasound waves consist of a cyclic succession of expansion (rarefaction) and compression phases imparted by mechanical vibration. Compression cycles exert a positive pressure and push the liquid molecules together, while expansion cycles exert a negative pressure and pull the molecules apart. This causes cavitation and bubble collapse process. In the presence of the small solid surfaces (composite reactions), the collapse of bubbles near the solid surface leads to formation of microjets which improve the mass transfer and speed up the transport process. For the time being cavitation bubble collapse, the temperature and pressure inside the bubble reach greater than $5000 \mathrm{~K}$ and $1000 \mathrm{~atm}$ [3]. Ultrasounds have the potential to influence the activity of the enzymatic reaction, if the energy input is not high enough to inactivate the enzyme. Ultrasounds (US) having frequencies ranging from $20 \mathrm{kHz}$ to $5 \mathrm{MHz}$ are a type of mechanical energy which do not shows ionizing radiation properties [4]. Earlier, ultrasound having frequency above $30 \mathrm{kHz}$ has been used as a method of enzyme inactivation to prevent the nutritive value of fruits and vegetables from undesirable effects (browning and off flavour) but recently, it has been found that activity of enzyme can be enhanced under milder condition of ultrasound irradiation. Enzymes are very sensitive to ultrasound treatment. An enzyme's catalytic activity is affected due to the continuous generation of waves and cavitation bubbles that leads to alteration in the loop and domain regions of enzyme [1].

Ultrasonic reactor is a system used for production of ultrasound. They are employed for various applications because of its special features. The occurrence of cavitation and acoustic streaming is the most important feature of ultrasonic reactors. Acoustic streaming causes physical effects 
and also enhances chemical processing limited by mass transfer. Sonochemical reactors are applied in various chemical and physical processes such as biotechnology, chemical synthesis, wastewater treatment, polymers degradation, waste water treatment, extraction, emulsification, crystallization, petrochemical industries and leaching, etc. These reactors are very sensitive and unresistant to operational parameters. The proper knowledge and understanding on physical and chemical phenomena is required to control the operational parameters [5].

Over the past years, it has been found lots of review papers on application of ultrasound in chemical engineering, textile engineering, medicine, etc. However, very few review literatures have given attention on use of ultrasound in bioprocess and biotechnology. A considerable overview of the manner in which ultrasound affects biotechnological process was published by Sinisterra (1992) in his review "Application of ultrasound to biotechnology ". However, due to unavailability of much information at that time, the reviewer has presented a number of unpublished examples of his own research [6]. In 2003, Chisti reviewed on the reactions catalyzed by live cells using ultrasound and concentrated totally on enhancing effect of ultrasonic waves on live biological system and the design requirements for ultrasonic reactors [7]. Rokhina et al. gave a review on the use of low frequency ultrasound in biotechnology and focused mainly in areas where sonochemistry can be profitably connected with biotechnology [2]. Recently, a mini review of the ultrasound assisted intensification of enzyme activity has been reviewed (Nadar et al., 2017). The authors have presented an overview of influence of ultrasonic parameters on enzyme activity and techniques for immobilization of ultrasound irradiated enzyme [1].

The present review article will provide a deep analysis of factors and recent development associated to intensification of enzymatic reaction using ultrasound. Also, it will briefly discuss the mechanism of intensification of enzymatic activity and in-depth knowledge about ultrasonic reactors and their types. At last, ultrasonic and enzymatic factors that affect the enzyme activity will be discussed. This review will provide all the aspects related to intensification of different enzymes at one platform. It will be advantageous in bioprocesses such as food processing, enzymatic conversions, chemical synthesis using enzyme and for the research scholar whoever going to start their project on intensification of enzymatic activity using ultrasound. 


\section{Mechanism of intensification of enzymatic reaction using ultrasound}

Introduction of ultrasound in liquid medium results in the generation of bubbles followed by collapse events. This event is termed as acoustic cavitation. The process of cavitation is represented in figure 1. The collapse of bubbles leads to formation of microjets which improve the mass transfer and speed up the transport of bulky enzyme molecule towards the solid or liquid interface where the real enzymatic reaction take place [8]. Ultrasound also changes the secondary structure of enzyme molecules that affects the enzyme activity. A study conducted on $\beta$-D- glucosidase showed the increase in $\alpha$-helices and decrease in $\beta$-folds and asymmetrical $\beta$ fold of $\beta$-D-Glucosidase on ultrasound application results in rapid activation of enzyme [9]. Moreover, cavitation creates a hydrodynamic shear force in the liquid medium due to the quick breakdown of microbubbles, which helps in the deterioration of large materials into small particles, in this manner fundamentally expanding the surface zone for enzymatic attack.

During sonication, formation of free radicals such as atomic hydrogen and hydroxyl group due to sonolysis of water, superoxides and hydrogen peroxide also takes place. These free radicals interact with amino acid residues of the enzymes and damages the complex structures of an enzyme. However, the probability of enzyme molecules to interact with these free radicals is very low. Ultrasonic shockwaves produced due to sudden collapse of microbubbles also stabilizes the enzyme by preventing the agglomeration of enzyme in solution [10].
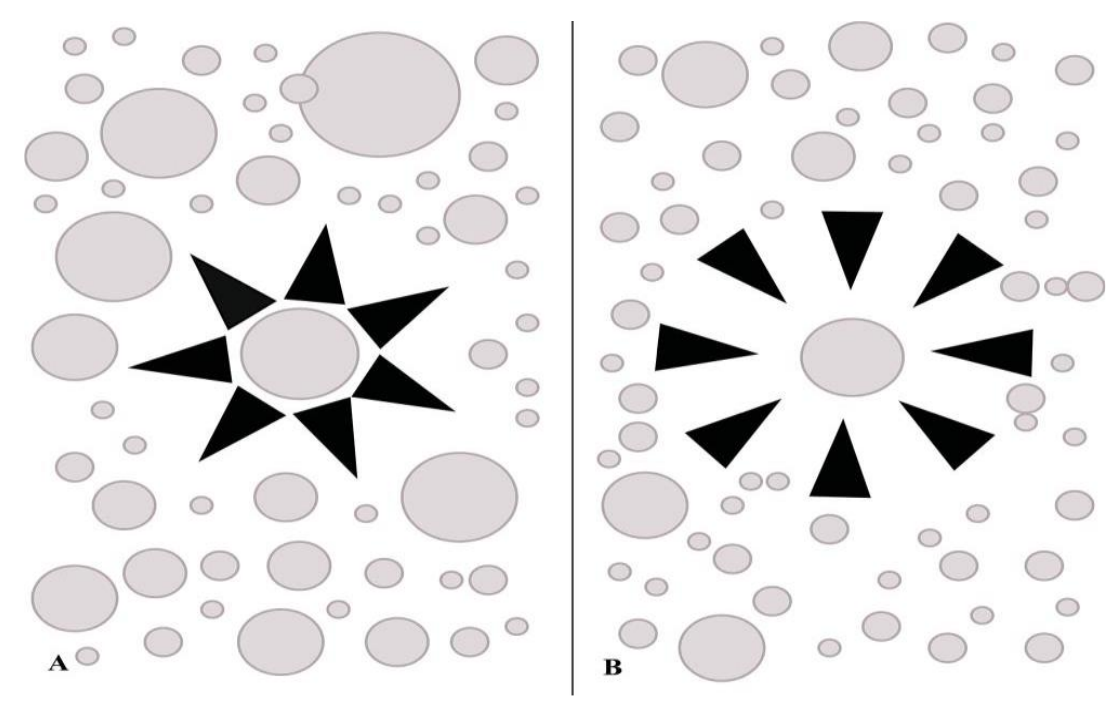

Fig. 1. Cavitation bubble formation. [A] Rarefaction [B] Compression [11] 


\section{Ultrasonic reactors for intensification of enzymatic reaction}

Introduction: It is a system that has capability to transform electrical energy into ultrasonic vibrations. Generator, transducer and the application system are the major parts of ultrasonic reactor. Generator is the source of energy and the transducers converts the energy produced by generator into mechanical energy in the form of vibrations [12]. Magneto-strictive and piezoelectric transducers are most common type of transducers used in ultrasonic reactors. Magneto-strictive transducers are made from high strength metallic alloys and they are very stable, consistent and resistant to degradation. However, piezoelectric transducers are most commonly used, because of their high energy transfer efficiency than magneto-strictive transducers [10]. Reactor efficiency can be enhanced by choosing an appropriate type of transducer and having sufficient knowledge about number of transducers and their position in the reactor. Use of multiple transducers in the reactor helps to create a uniform distribution. Multiple transducers also provide better control of hydrodynamic conditions and mixing and the ability to use multiple frequencies. The use of ultrasonic waves always heats the solution and reactor during use. Therefore, the cooling of the system should also be carefully planned [13].

Types of ultrasonic reactors: Ultrasonic horns and ultrasonic baths are the most common type of ultrasonic reactor used for enzymatic intensification reactions at laboratory scale. In the ultrasonic horn reactor, the transmitting element which submerges in the liquid medium consists of a horn linked to the transducer. Ultrasonic horn is a immersion type of reactor in which the ultrasonic waves are directly transmitted into the medium by vibrating horn [14]. Cavitational effects are very high close to the vibrating horn and decreases exponentially on moving away from the vibrating surface. The efficiency of horn type reactors is very low for large scale operations as compared to reactors based on multiple transducers. Additionally, erosion of horn tip due to high power applied to the horn and particle peeling increases the chances of stress induced fatigue failure [15]. However, certain modifications are made in the shape and position of horn by some researchers to increase the sonochemical yield. Barbell-shaped horn, Donut shaped ultrasonic horn, Concentrator horn, Telsonic horn are the examples of modified form of ultrasonic horn. However, they are not powerful enough for scale up prospects because their capacity for transmission of irradiation into the large tank volume seems slightly weak [10]. Horns can also be used longitudinally in the vessel for different applications. The longitudinal 
horns usually have higher surface area of irradiation in the medium and the magnitude of energy efficiency in this type of ultrasonic is higher than the conventional one. Moreover, the broad irradiation area of longitudinal ultrasonic horns leads to uniform distribution of cavitational activity in the whole reactor volume which can be more beneficial in pilot scale in comparison with simple ultrasonic horns [13].

Ultrasonic baths are also commonly used ultrasonic reactors in which transducers are attached at the bottom of the reactor and ultrasound irradiations are transmitted into the system indirectly. Cavitational intensity of ultrasonic baths are less as compared to ultrasonic horns because they are indirect irradiation based ultrasonic reactors. They are generally applied where specific ultrasonic intensity is not required. The maximum operating capacity of the ultrasonic bath is about 3 litres, but baths with higher capacity (up to 1000 litre) is also possible with some modification in terms of larger number of transducers in different configurations. Moreover, ultrasonic bath-type of reactors are suitable for laboratory to large-scale operations, but there is a problem on the number of transducers that can be attached in a system for a large-scale operation [5]. Dual frequency flow cell and triple-frequency flow-cell-type reactors are the reactors based on the use of multiple transducers. They are very much effective in continuous operations. These reactors produce a beat frequency inside a chamber that continually oscillates within reactor chamber and ensures constant processing. There are two sets of magneto-strictive transducers (i.e., three in each side) attached on the two opposite walls in dual frequency flow cell. In case of triple frequency flow cell, there are three transducers attached in each set per side and having equal power dissipation per side. The use of multiple transducers is helpful in concentrating the ultrasonic waves towards the central zone of vessel $[5,15]$.

\begin{tabular}{|l|c|}
\hline Type of reactor & Cavitational yield (mol/W) \\
\hline Ultrasonic horn & 0.0005 \\
\hline Ultrasonic bath & 0.01 \\
\hline Dual frequency flow cell & 0.011 \\
\hline Triple frequency flow cell & 0.018 \\
\hline Longitudinally horn reactor & 0.077 \\
\hline
\end{tabular}

Table 1. Comparison of cavitational yield in ultrasonic reactors [5] 


\section{Factors affecting enzymatic reactions}

Ultrasonic frequency: The frequency of an ultrasound is an important influential factor for enzymatic reactions. The ultrasounds frequency has a direct influence on the cavitation bubble collapse. Ultrasonic irradiation of enzyme at optimum frequency causes conformational changes in protein structure of enzyme which leads to increment in enzyme activity. A research was conducted on the effect of ultrasound frequency on cellulase activity. The researchers have found an increment in catalytic activity of immobilized cellulose by $6.56,14.79,17.85$ and $10.45 \%$ at frequencies of 18,20, 24 and $26 \mathrm{kHz}$, respectively. The increment in catalytic activity was due to the ability of ultrasound to increase the surface area of immobilized enzyme molecules. However, a decrement of $1.02 \%$ at $29 \mathrm{kHz}$ was found in catalytic activity of enzyme molecule due to rapid collapse of cavitation bubbles at higher frequencies which leads formation of excessive heat [16]. In another research, the effect of ultrasound frequency on alkaline protease catalytic activity was analyzed by exposing enzyme at three different frequencies 40, 80, and 100 $\mathrm{kHz}$. The yields under ultrasound were better than that of shaking and at $80 \mathrm{kHz}$, highest yield was found. Thus, ultrasound irradiation with optimum frequency is needed during treating enzyme to bring better results [17].

Ultrasonic power or intensity: Ultrasonic intensity is an important factor which plays major role in enhancing or inhibitory effects of ultrasound on enzyme activity. Yujing et al. (2019) conducted an experiment on the effect of ultrasonic intensity on $\beta$-d-glucosidase catalytic activity and found that the activity was increased with increasing ultrasonic intensity up to 181.53 $\mathrm{W} / \mathrm{cm}^{2}$. However, its activity started decreasing after $181.53 \mathrm{~W} / \mathrm{cm}^{2}$. The results showed that use of low intensity ultrasound creates cavitation and mechanical oscillation which leads to conformational changes in enzyme and increase its contact with the substrate. Under the condition of high intensity ultrasound, inhibition of enzyme activity occurs due to interaction of generated free radicals with protein backbone [9]. In another study, the effect of ultrasound intensity on cellulase activity was explored by irradiating an enzyme to various intensities and maximum activity was observed at $17.33 \mathrm{~W} / \mathrm{cm}^{2}$. The explanation for this is, low intensity ultrasound breaks the weak interactions such as hydrogen bonds or Van der Waals forces which bring the conformational alteration in active site of enzyme [18]. 
Duty cycle: Duty cycle is another important parameter of ultrasound that affects the enzyme activity. The use of a duty cycle is more economical than continuous energy application. It keeps the temperature of the reaction sample at optimum levels and protects the enzyme from denaturation. It also helps to control irradiation time of ultrasound on enzyme and reduction in energy consumption [19]. A study was conducted on $\beta$-d-glucosidase for evaluation of effect of duty cycle on its catalytic activity. The results indicated that the activity was enhanced when duty cycle was between $33.33 \%$ to $40 \%$. However, a slight decrease in yield was found when duty cycle ranged from $40 \%$ to $100 \%$. The reason for the decrease in yield at higher duty cycle was due to extensive cavitation which affect the active conformation of enzyme. On the other side, a lower duty cycle seems to be a better choice for the activation of enzyme [9]. In another study on lipase at different duty cycle showed the maximum enzyme activity at $66.67 \%$. The reason for maximum activity at $66.67 \%$ might be due to proper application of impulsive forces on enzyme was given by authors [20]

Concentration of enzyme: Concentration of enzyme plays a critical role in analyzing the effects of ultrasound irradiation. At very low concentration of enzyme, the probability of enzyme molecules to effectively interact with micro streams generated due to acoustic cavitation is very less. Conversely, above the optimum concentration, excessive enzyme molecules impede the energy-transfer process, thereby decreasing the available energy for cavitational phenomenon. An excess enzyme molecule also increases the probability of forming aggregates of enzymes due to cavitation, which results in a lower degree of intensification. Enzyme kinetics also indicated the decrease in rate of reaction due to competition for substrate by enzyme molecules at high concentration of enzyme [20].

pH and temperature: $\mathrm{pH}$ and temperature both plays an important role in determining the effects of ultrasound irradiation. Higher and lower $\mathrm{pH}$ and temperature can lead to partial or complete inhibition of enzyme catalytic activity. Wei-ming Wang et al. (2012) investigated the effect of $\mathrm{pH}$ on amylase activity applied for desizing of cotton fabric. The authors have found that desizing percentage values significantly increased with $\mathrm{pH}$ value increased upto 6 . However, antagonistic effects have been found on catalytic activity of amylase above pH value of 6[21]. Souza et al. studied the effects of temperature on the activity of alpha amylase on ultrasound treatment. They found three times increase in catalytic activity of alpha amylase for temperature increase up to $40 \%$ on ultrasonic irradiation. The results indicated that the cavitation is higher at 
lower temperature and optimum temperature range is required to break strong interaction such as hydrogen bonding, dipole attractions and Vander Waal forces between the substrate and active sites on the enzyme [22].

\section{Conclusion}

In summary, Low intensity ultrasound can be used as a tool for intensification of enzymatic reaction. It is very effective in activation of enzyme by changing the structural conformation of active site and also effective in improving the stability of enzyme and kinetic factors. Cavitation phenomenon from ultrasound is required to attain higher reaction rates. Choosing an appropriate reactor is a critical task for specific enzymatic reactions. An ultrasonic reactor appears to be very effective for enzymatic intensification reactions. It is economically beneficial due to their special features such as acoustic cavitation and acoustics streaming. Parameters such as ultrasonic frequency, ultrasonic intensity, duty cycle, temperature, $\mathrm{pH}$ and treatment time determine the activation or inhibition of enzyme. Controlling these parameters and maintain uniform distribution of cavitational activity in reactor is one of the most important challenges in scale up ultrasonic reactors. There is lack of proper information about enzyme working environment. Therefore, it is necessary to collect complete information on ultrasonic effects under various experimental conditions. It will be helpful in developing mathematical model and easy prediction of changes in the efficiency of the process.

\section{Acknowledgement}

We wish to show my appreciation to Institute of Chemical Technology, Mumbai for providing us the right environment to write this review.

\section{References}

1. Nadar, S. S., \& Rathod, V. K. (2017). Ultrasound assisted intensification of enzyme activity and its properties: a mini-review. World Journal of Microbiology and Biotechnology, 33(9), 170.

2. Rokhina, E. V., Lens, P., \& Virkutyte, J. (2009). Low-frequency ultrasound in biotechnology: state of the art. Trends in biotechnology, 27(5), 298-306. 
3. Bhangu, S. K., \& Ashokkumar, M. (2017). Theory of sonochemistry. In Sonochemistry (pp. 1-28). Springer, Cham.

4. Vartolomei, A., Calinescu, I., Vinatoru, M., \& Gavrila, A. I. (2019). Intensification of the Enzymatic Esterification Process by Ultrasounds. REVISTA DE CHIMIE, 70(1), 41-44.

5. Asgharzadehahmadi, S., Raman, A. A. A., Parthasarathy, R., \& Sajjadi, B. (2016). Sonochemical reactors: Review on features, advantages and limitations. Renewable and Sustainable Energy Reviews, 63, 302-314.

6. Sinisterra, J. V. (1992). Application of ultrasound to biotechnology: an overview. Ultrasonics, 30(3), 180-185.

7. Chisti, Y. (2003). Sonobioreactors: using ultrasound for enhanced microbial productivity. TRENDS in Biotechnology, 21(2), 89-93.

8. Yachmenev, V. G., Bertoniere, N. R., \& Blanchard, E. J. (2002). Intensification of the bio-processing of cotton textiles by combined enzyme/ultrasound treatment. Journal of Chemical Technology \& Biotechnology, 77(5), 559-567.

9. Sun, Y., Zeng, L., Xue, Y., Yang, T., Cheng, Z., \& Sun, P. (2019). Effects of power ultrasound on the activity and structure of $\beta$-D-glucosidase with potentially aroma-enhancing capability. Food science \& nutrition, 7(6), 2043-2049.

10. Subhedar, P. B., \& Gogate, P. R. (2013). Intensification of enzymatic hydrolysis of lignocellulose using ultrasound for efficient bioethanol production: a review. Industrial \& Engineering Chemistry Research, 52(34), 11816-11828.

11. Islam, M. N., Zhang, M., \& Adhikari, B. (2014). The inactivation of enzymes by ultrasound-a review of potential mechanisms. Food Reviews International, 30(1), 1-21.

12. Cárcel, J. A., García-Pérez, J. V., Benedito, J., \& Mulet, A. (2012). Food process innovation through new technologies: Use of ultrasound. Journal of Food Engineering, 110(2), 200-207.

13. Gogate, P. R., \& Patil, P. N. (2017). Sonochemical reactors. In Sonochemistry (pp. 255281). Springer, Cham

14. Chemat, F., \& Khan, M. K. (2011). Applications of ultrasound in food technology: processing, preservation and extraction. Ultrasonics sonochemistry, 18(4), 813-835

15. Gogate, P. R., \& Kabadi, A. M. (2009). A review of applications of cavitation in biochemical engineering/biotechnology. Biochemical Engineering Journal, 44(1), 60-72 
16. Wang Z, Lin X, Li P, Zhang J, Wang S, Ma H (2012) Effects of low intensity ultrasound on cellulase pretreatment. Bioresour Technol 117:222-227

17. Xiao, Y., Yang, L., Mao, P., Zhao, Z., \& Lin, X. (2011). Ultrasound-promoted enzymatic synthesis of troxerutin esters in nonaqueous solvents. Ultrasonics sonochemistry, 18(1), 303-309.

18. Subhedar, P. B., \& Gogate, P. R. (2014). Enhancing the activity of cellulase enzyme using ultrasonic irradiations. Journal of Molecular Catalysis B: Enzymatic, 101, 108-114.

19. Freitas, V. O. D., Matte, C. R., Poppe, J. K., Rodrigues, R. C., \& Ayub, M. A. (2019). Ultrasound-assisted transesterification of soybean oil using combi-lipase biocatalysts. Brazilian Journal of Chemical Engineering, 36(2), 995-1005.

20. Jadhav SH, Gogate PR (2014) Intensification in the activity of lipase enzyme using ultrasonic irradiation and stability studies. Ind Eng Chem Res 53:1377-1385

21. Wang, W., Yu, B., \& Zhong, C. (2012). Use of ultrasonic energy in the enzymatic desizing of cotton fabric. Journal of Cleaner Production, 33, 179-182.

22. Souza M, Mezadri ET, Zimmerman E et al (2013) Evaluation of activity of a commercial amylase under ultrasound-assisted irradiation. UltrasonSonochem 20:89-94 